\title{
Quorum Sensing Materials as New Ligand for Palladium Catalyzed Migita-Kosugi-Stille Polycondensation
}

\author{
Aohan Wang ${ }^{1}$, Masanori Toyofuku ${ }^{2}$, Nobuhiko Nomura ${ }^{2}$, Hiromasa Goto ${ }^{1, *}$ \\ ${ }^{1}$ Division of Materials Science, Faculty of Pure and Applied Sciences, University of Tsukuba, \\ Tsukuba, Ibaraki 305-8573, Japan \\ ${ }^{2}$ Graduate School of Life and Environmental Sciences, University of Tsukuba, University of Tsukuba, \\ Tsukuba, Ibaraki 305-8572, Japan \\ *E-mail address: gotoh@ims.tsukuba.ac.jp
}

\begin{abstract}
Signal molecules for quorum sensing are employed for ligands to palladium. The complex can be used as a catalyst. Migita-Kosugi-Stille coupling reactions are driven by using a series of N-(3oxo-acyl)-L-homoserine lactones as ligands coordinating with Pd. Catalysis of [Pd-(signal molecules for quorum sensing), or $\mathrm{Pd} / \mathrm{QS}$ ] allows production of conjugated polymers with light-emitting functions.
\end{abstract}

Keywords: signal molecules; quorum sensing; Migita-Kosugi-Stille coupling; $\pi$-conjugated polymers

\section{INTRODUCTION}

Bacteria communicate with one another by using small chemical molecules [1]. This process, known as quorum sensing, allows bacteria to regulate their gene expression in response to their population [2,3]. In addition to the biological functions, quorum sensing signals has chemical functions that remains poorly studied [4-6]. One of the most widely observed quorum-sensing signals among Gram-negative bacteria is acyl homoserine lactones (AHLs). A group of AHLs have carbonyl groups in the chemical structure that may interact with metals to form complexes [7]. In particular, 3-oxo-CR-homoserine lactones (3-oxo-CRHSL; where $R=$ carbon number), possess a $\beta$-diketon group:<smiles>CC(=O)CC(C)=O</smiles>

$\beta$-diketon 
The $\beta$-diketon group can coordinate with $\mathrm{Fe}, \mathrm{Pd}[7,8]$, and $\mathrm{Ni}$ to form metal complexes. These $\beta$-diketon metal complexes such as Metal-acetyl acetonate complexes can be used as a Ziegler-Natta catalytic system. For instance, iron acetylacetonate was employed as a cocatalyst for the synthesis of polyacetylene derivatives [4]. The presence of $\beta$-diketon in 3 oxo-CR-HSLs suggest that these quorum sensing molecules, in the form of [Pd(II)] complex, can perform catalysis for chemical reactions such as coupling reactions (Miyaura-Suzuki coupling [9], Migita-Kosugi-Stille coupling [10,11], Kumada coupling [12]).

Here we demonstrate that the quorum sensing signaling molecule, together with $[\mathrm{Pd}(\mathrm{II})]$, can be utilized for chemical reactions. In this report, we conduct Migita-KosugiStille coupling reactions to construct $\pi$-conjugated polymers with the aid of [Pd-(signal molecules for quorum sensing), $\mathrm{Pd} / \mathrm{QS}]$. This is the first report of polymerisations by using complexes of metal-(signal materials for quorum sensing) $[13,14]$.

\section{EXPERIMENTAL}

\section{1. Chemicals}

We employed the substances, butyl-homoserine lactone (C4-HSL), $N$-(3-oxohexanoyl)L-homoserine lactone (3-oxo-C6-HSL), $N$-(3-oxododecanoyl)-L-homoserine lactone (3-oxo$\mathrm{C} 12-\mathrm{HSL})$ and $\mathrm{N}$-(3-oxohexadecanoyl)-L-homoserine lactone (3-oxo-C16-HSL). Chemical structures of the signal substances for quorum sensing for ligands to Pd are shown in Fig. 1.

\section{1. 1. Preparation of reference sample (poly1)}

2,5-Dibromobenzene-1-dodecyl ester and bistrimethylstannylthiophene were stirred in tetrahydrofuran (THF) for $48 \mathrm{~h}$ in the existence of $\mathrm{PdCl}_{2}$ to yield red powder (poly1). This compound synthesised without the quorum sensing substances is a reference sample to the polymers prepared with $\mathrm{Pd} / \mathrm{QS}$.<smiles>CCCC(=O)NC1CCOC1=O</smiles>

C4-HSL<smiles>CCCC(=O)CC(=O)NC1CCOC1=O</smiles>

3-OXO-C6-HSL<smiles>CCCCCCCCCC(=O)CC(=O)NC1CCOC1=O</smiles>

3-OXO-C12-HSL<smiles>CCCCCCCCCCCCCC(=O)CC(=O)NC1CCOC1=O</smiles>

Figure 1. Chemical structures of quorum sensing substances. 


\section{1. 2. Polymerisation in the presence of $P d / Q S$}

A solution of the quorum sensing molecule (C4-HSL, 3-oxo-C6-HSL, 3-oxo-C12-HSL, or 3-oxo-C16-HSL) and $\mathrm{PdCl}_{2}$ was refluxed in THF for $1 \mathrm{~h}$, presumably resulting in coordination with $\mathrm{Pd}$.

Then, 2,5-dibromobenzene-1-dodecyl ester and bistrimethylstannylthiophene as monomers were added to the solution and refluxed. After $24 \mathrm{~h}$, the solution was poured into a large volume of methanol and stirred for $24 \mathrm{~h}$. Filtration followed by drying in vacuum yielded a red powder as the desired polymer. The polymer thus obtained is abbreviated as poly2(C4), prepared by using C4-HSL as a cocatalyst, poly3(C6) with 3-oxo-C12-HSL as a cocatalyst, poly4(C12) with 3 -oxo-C12-HSL as a cocatalyst, and poly5(C16) with 3-oxoC16-HSL as a cocatalyst (Scheme 1).

Quantities of the chemicals employed in the polymerisations and synthetic yield of the polymers are summarized in Table 1. Molecular weight measurements for the polymers were carried out using gel permeation chromatography (GPC) with THF as an eluent. Numberaverage-molecular weights $\left(M_{n}\right)$ and weight-average-molecular weights $\left(M_{w}\right)$ of poly5(C16) were found to be 2,600 and 3,200, respectively.

Dispersion of the polymer was 1.2. Molecular weights of poly1 could not be obtained using the GPC due to poor solubility in THF, indicating poly1 is cross-linked. Side-reactions at the $\beta$-positions of the thiophene unit can be occurred by the $\mathrm{PdCl}_{2}$ driven oxidative reaction to form cross-link. Poly2(C4), poly3(C6) and poly4(C12) are partly soluble in THF. $M_{n}$ of the soluble part of these polymers was ca. 1,000.

Table 1. Polymerisation ${ }^{\mathrm{a}}$ and Synthetic yield of the Polymers.

\begin{tabular}{lcc}
\hline Polymer & QS $^{\mathrm{b}}$ & $\mathrm{Y}(\mathrm{mg})$ \\
\hline Poly1 & - & 8.0 \\
\hline Poly2(C4) & C4-HSL & 6.7 \\
Poly3(C6) & 3-oxo-C6-HSL & 2.4 \\
Poly4(C12) & 3-oxo-C12-HSL & 10.7 \\
Poly5(C16) & 3oxo-C16-HSL & 15.1 \\
\hline${ }^{\mathrm{a} Q u a n t i t y ~ u s e d: ~}$ PdCl $_{2}$ & $($ catalyst, 1 mg), QS (co-catalyst, 8 \\
$\mathrm{mg})$, DBB11 (monomer, 100 mg), Th-TMS & (monomer, 100 \\
$\mathrm{mg})$.
\end{tabular}<smiles>CCCCCOC(=O)c1cc(Br)ccc1Br</smiles>

: DBB 11,<smiles>C[Sn](C)(C)c1ccc([Sn](C)(C)C)s1</smiles>

${ }^{\mathrm{b}}$ Quorum sensing substance 


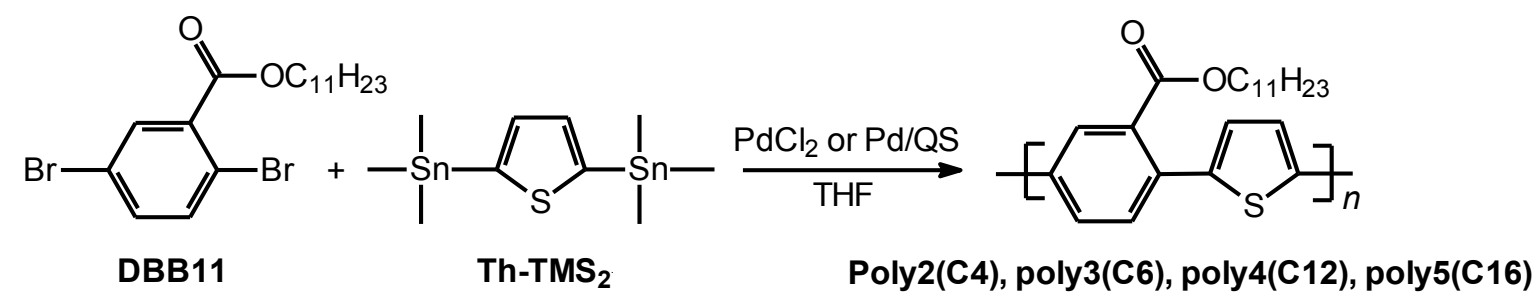

Scheme 1. Synthesis of polymers. $\mathrm{Pd} / \mathrm{QS}=[\mathrm{Pd}-($ signal molecules for quorum sensing substance $(\mathrm{QS})]$; THF $=$ tetrahydrofuran.

\section{RESULTS AND DISCUSSION}

\section{1. Infrared absorption}

Infrared (IR) optical absorption spectroscopy measurements were carried using the $\mathrm{KBr}$ method (Fig. 2). The absorption corresponding to the $\mathrm{CH}$ out-of-plane bending of aromatic rings was observed at $807 \mathrm{~cm}^{-1}$. An absorption band at $3438 \mathrm{~cm}^{-1}$ can be attributed to $\mathrm{OH}$ stretching vibration. The absorption band at $1726 \mathrm{~cm}^{-1}$ can be ascribed to -CO-O- stretching of the ester group in the side chains. Stretching at $1240 \mathrm{~cm}^{-1}$ is due to a C-O-C asymmetric stretching vibration. An absorption band at $2932 \mathrm{~cm}^{-1}$ is likely due to stretching vibrations of the alkyl chains $\left(v \mathrm{CH}_{2}, v \mathrm{CH}_{3}\right)$. The characteristic absorption band at $697 \mathrm{~cm}^{-1}$ is likely due to $\delta \mathrm{CH}$ of the thiophene ring.

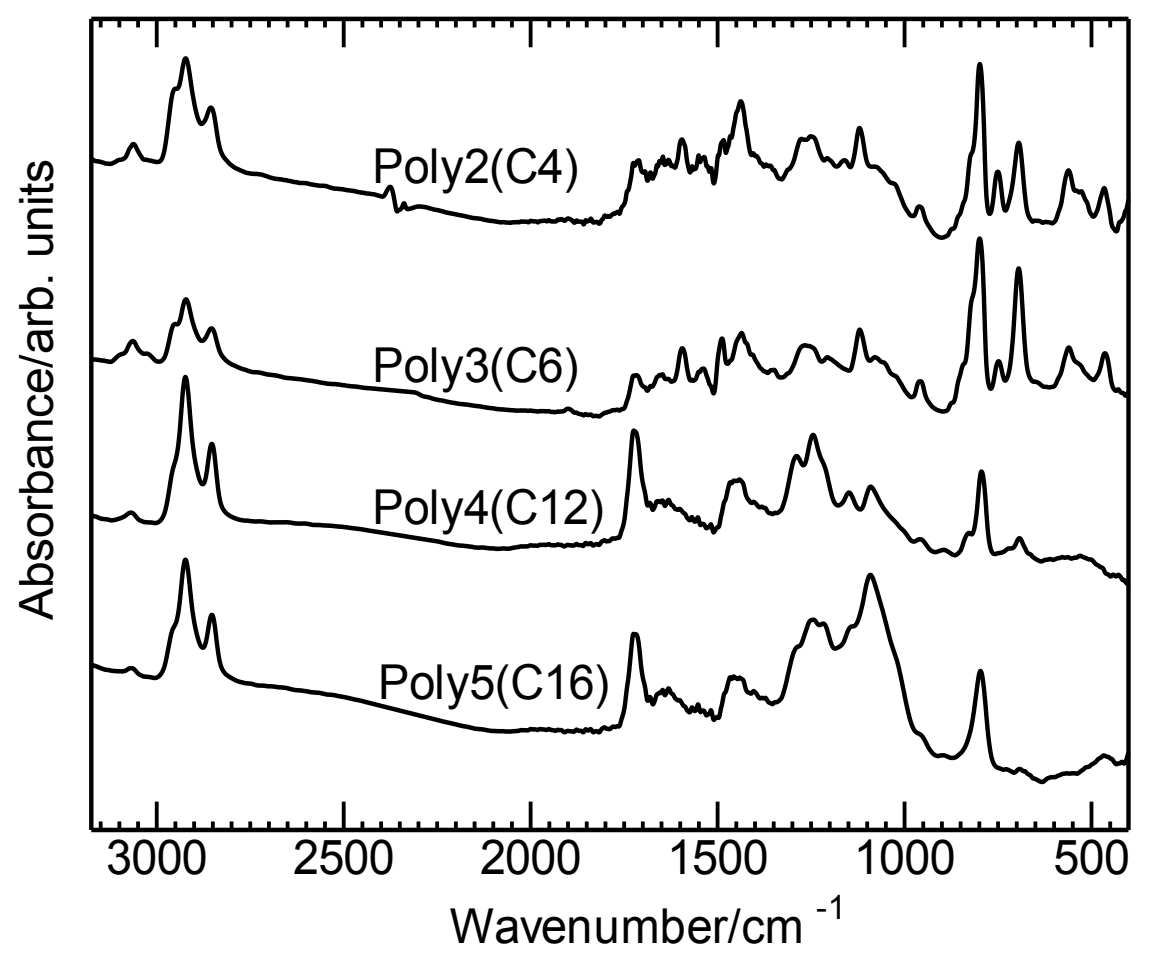

Figure 2. Infrared absorption spectra of the polymers prepared with the aid of $\mathrm{Pd} / \mathrm{QS}$. 
However the IR spectrum for polyl showed no clear absorption bands due to the alkyl chains $\left(v_{\mathrm{CH}_{2}}, v \mathrm{CH}_{3}\right)$, indicating occurrence of side reactions, such as cross-linking. The QS substances can improve solubility of the $\mathrm{PdCl}_{2}$ by coordination to form the complex. The soluble complex in THF affords effective catalysis for the polycondensation reaction. On the other hand, inhomogeneous polymerisation by $\mathrm{PdCl}_{2}$ with poor solubility resulted in crosslinked polymers.

\section{2. UV-Vis absorption}

UV-Vis optical absorption spectra of the polymers were obtained in THF solution (Fig. 3). Poly2(C4) and poly3(C6) showed absorption maxima at $392 \mathrm{~nm}$ due to $\pi-\pi *$ transition of the main chain. Poly4(C12) and poly5(C16) display an absorption maximum at $423 \mathrm{~nm}$, which is a longer wavelength than that of poly2(C4) and poly3(C6), indicating that the poly4(C12) and poly5(C16) have long effective conjugation lengths. This may be due to the quorum sensing materials with long alkyl chain aid catalytic activity of Pd providing good coplanarity of the polymers. An absorption spectrum of polyl could not be obtained due to insolubility.

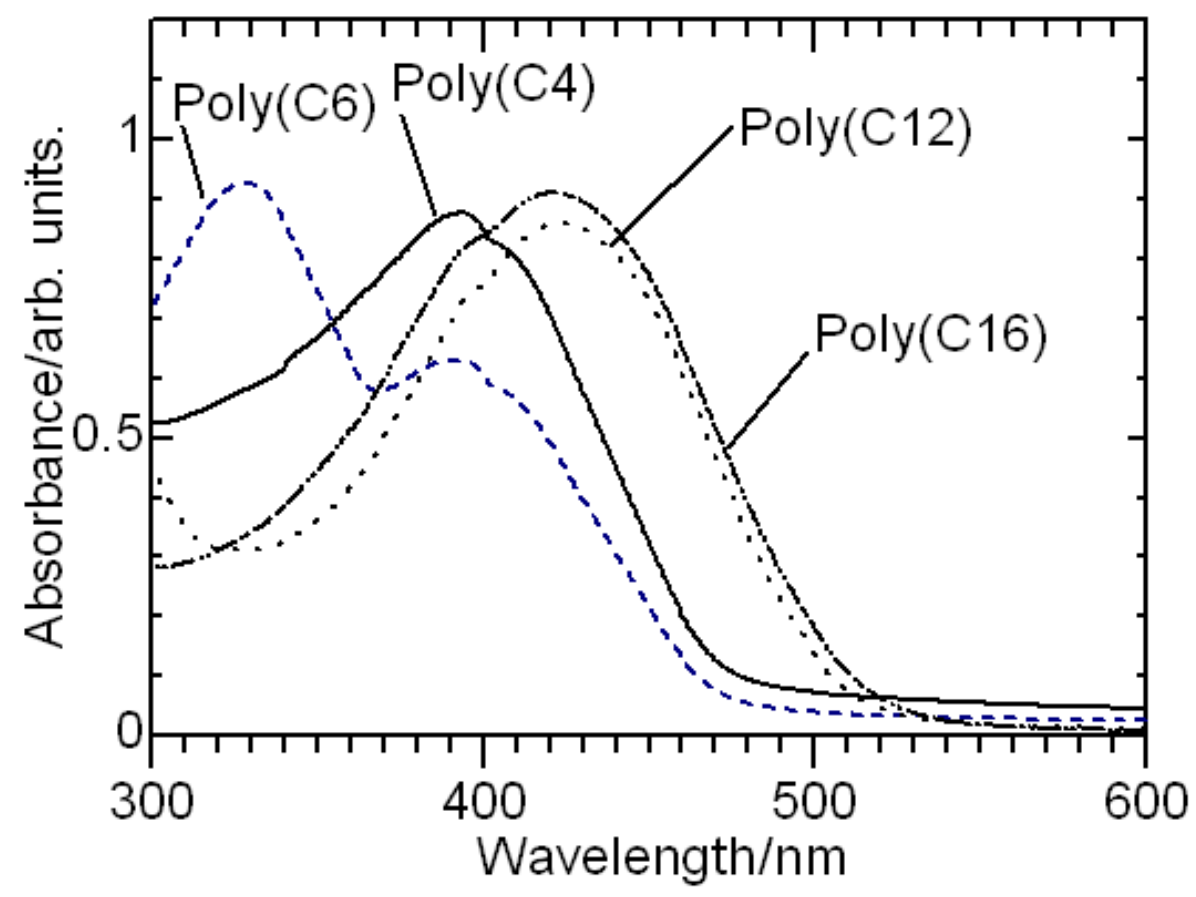

Figure 3. UV-Vis optical absorption spectra of polymers prepared with the aid of $\mathrm{Pd} / \mathrm{QS}$.

\section{3. Photoluminescence}

Figure 4 displays photoluminescence (PL) spectroscopy measurements. Poly2(C4) and poly3(C6) show PL maxima at $483 \mathrm{~nm}$, while poly4(C12) $530 \mathrm{~nm}$ and poly5(C16) $540 \mathrm{~nm}$. These results are well matched with the results of the absorption spectroscopy measurements. The polymers prepared by using Pd-quorum sensing materials show light emission derived from the sequence of $\pi$-conjugation systems. 


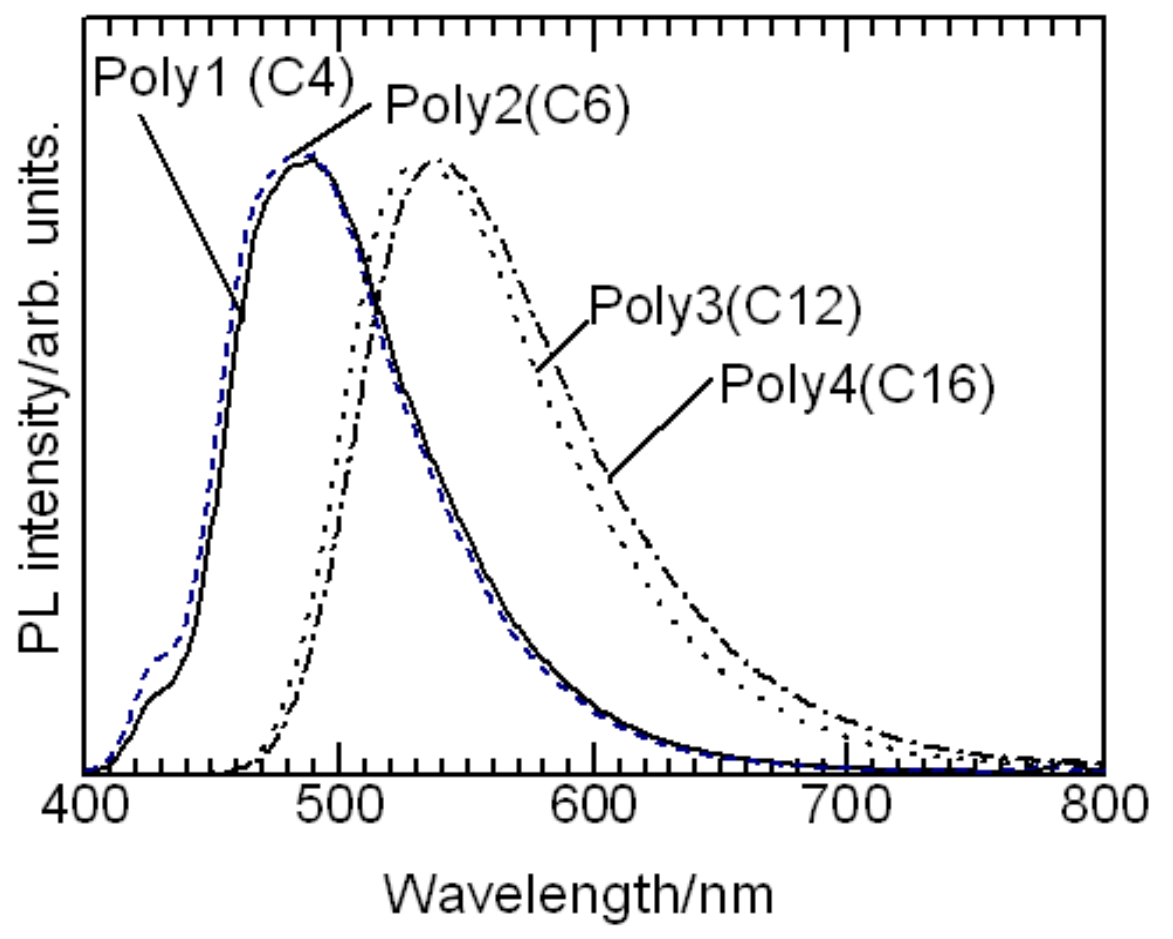

Figure 4. Photoluminescence spectra of polymers prepared with the aid of the $\mathrm{Pd} / \mathrm{QS}$.

\section{4. X-ray Photoelectron Spectroscopy (XPS)}

XPS measurements evaluated that all of the polymers contain carbon atoms (75\%) and oxygen atoms $(20 \%)$, and trace amount of nitrogen detected at $2 \%$. Figure 5 displays the existence of 1s-orbital of carbon and oxygen at $284 \mathrm{eV}$ and $532 \mathrm{eV}$, respectively. A small peak at $164 \mathrm{eV}$ reveals the existence of sulfur in the polymers. No peaks are observed for nitrogen in the wide-scan measurement. We conclude that the polymers thus obtained with the aid of $\mathrm{Pd} / \mathrm{QS}$ are pure materials. Composition of each polymer was calculated by narrow scan measurements (Table 2).

Table 2. Atomic Composition of Polymers ${ }^{\mathrm{a}}$

\begin{tabular}{cccc}
\hline & $\mathrm{C}(\%)$ & $\mathrm{O}(\%)$ & $\mathrm{N}(\%)$ \\
\hline Poly2(C4) & 79.03 & 19.11 & 1.85 \\
Poly3(C6) & 79.25 & 18.55 & 2.21 \\
Poly4(C12) & 75.84 & 22.46 & 1.67 \\
Poly5(C16) & 73.08 & 25.72 & 1.20 \\
\hline
\end{tabular}

${ }^{\mathrm{a}}$ Composition is calculated by narrow-scan measurements. 


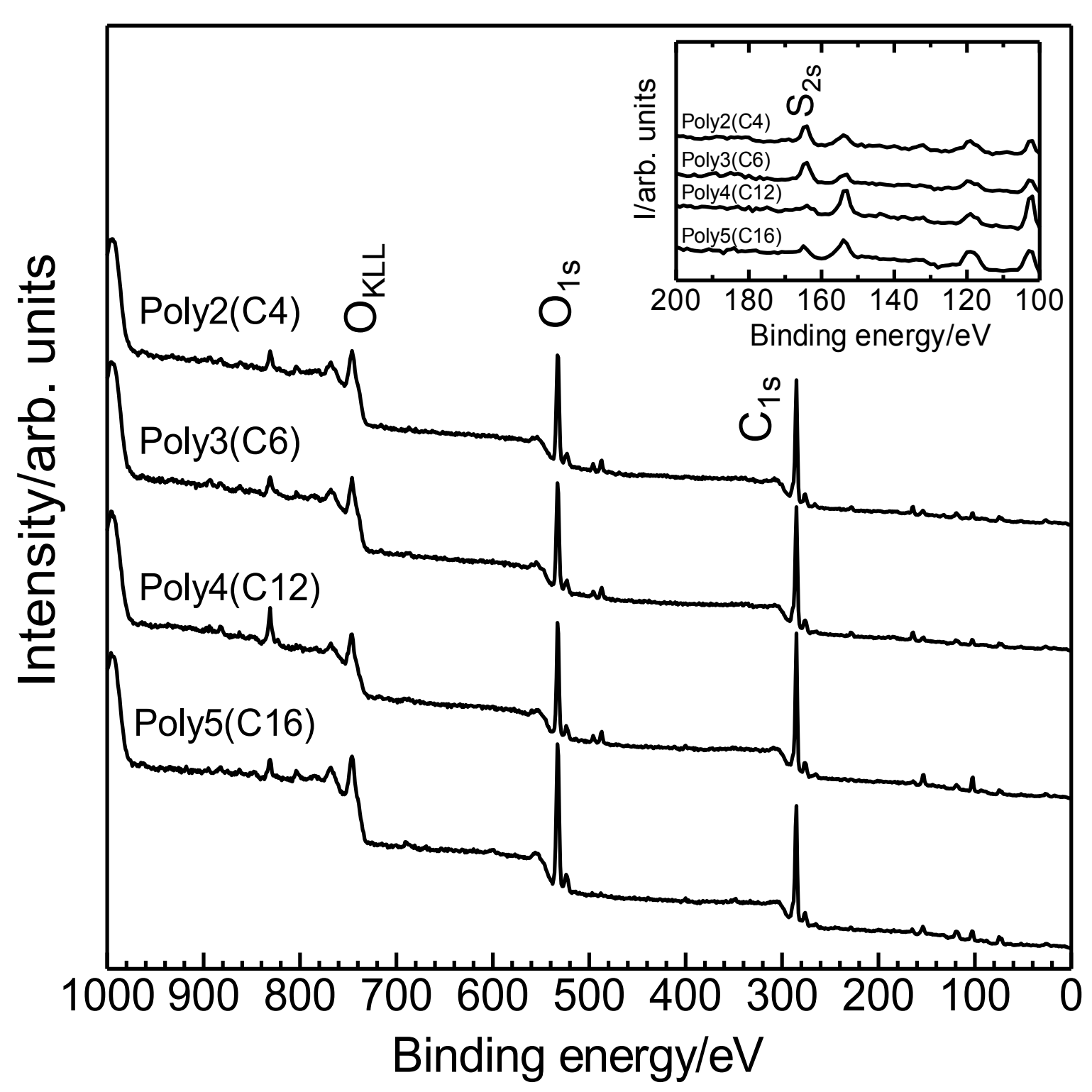

Figure 5. X-ray photoelectron spectra of polymers.

\section{CONCLUSIONS}

We have achieved polycondensation to obtain $\pi$-conjugated polymers by using a series of Pd- $\square$-diketons of signal materials for quorum sensing. Although the Migita-Kosugi-Stille type polycondensation with common acetyl acetonate compounds may be performed, significance of the present result is construction of $\pi$-conjugated sequences by using the signal materials for quorum sensing. The polymer synthesised without quorum sensing materials could not be detected using GPC because of the poor solubility. Long alkyl chains of quorum sensing materials may have good affinity with the monomers and polymer chains in the polymerisation process. The spectroscopy measurements confirmed the structures and 
the optical properties of the resultant polymers. The coordination system for sensing was applied for production of electro-optical functional polymers.

\section{Techniques}

Molecular weights of the polymers were determined by gel permeation chromatography (GPC) with MXED-D HPLC column (Polymer Laboratories), PU-980 HPLC pump (JASCO) and MD-815 multi-wavelength detector (JASCO), with tetrahydrofuran (THF) used as the solvent, with the instruments calibrated by polystyrene standard. Infrared (IR) spectroscopy was carried out with FT/IR-300 spectrometer (JASCO). Ultraviolet-visible (UV-Vis) absorption spectra and photoluminescence (PL) spectra of the polymers in chloroform were obtained with a V-630 UV-Vis optical absorption spectrometer (JASCO) and an F-4500 fluorescence spectrophotometer (Hitachi). XPS spectroscopy measurements were performed using a JPS-9010TR (JEOL).

\section{ACKNOWLEDGMENT}

The PL spectra were obtained on an F-4500 (Hitachi) at the University of Tsukuba and the OPEN FACILITY, Research Facility Center for Science and Technology, University of Tsukuba. We thank the Engineering Workshop of the University of Tsukuba for glasswork.

\section{References}

[1] C. Fuqua, E. P. Greenberg, Nature Reviews Molecular Cell Biology 3 (2002) 685-695.

[2] I. Sawada, H. Maseda, T.Nakae, H. Uchiyama, N. Nomura, Microbiology and Immunology 48 (2004) 435-439.

[3] H. Maseda, I. Sawada, K. Saito, H. Uchiyama, T. Nakae, N. Nomura, Antimicrobial Agents and Chemotherapy 48 (2004) 1320-1328.

[4] G. F. Kaufmann, et al., Proceedings of the National Academy of Sciences 102 (2005) 309-314.

[5] F. Bredenbruch, R. Geffers, M. Nimtz, J. Buer, S. Häussler, Environmental microbiology 8 (2006) 1318-1329.

[6] S. P. Digger, et al., Chemistry and Biology 14 (2007) 87-96.

[7] S. Baba, T. Ogura, S. Kawaguchi, Bulletin of the Chemical Society of Japan 47 (1974) 665-668.

[8] P. M. Maitlis, P. Espinet, M. J. H. Russell, Comprehensive Organometallic Chemistry 6 (1982) 233-242.

[9] N. Miyaura, K. Yamada, A. Suzuki, Tetrahedron Letters 36 (1979) 3437-3440.

[10] M. Kosugi, K. Sasazawa, Y. Shimizu, T. Migita, Chemistry Letters (1977) 301-302.

[11] J.K. Stille, Angewandte Chemie International Edition 25 (1986) 508-524.

[12] K. Tamao, Y. Kiso, K. Sumitani, M. Kumada, Journal of the American Chemical Society 94 (1972) 9268-9269. 
[13] Kuniharu Nakajima, Hiromasa Goto, International Letters of Chemistry, Physics and Astronomy 6 (2014) 33-38.

[14] Hiroki Hayashi, Kohsuke Kawabata, Hiromasa Goto, International Letters of Chemistry, Physics and Astronomy 8 (2014) 6-12.

( Received 03 April 2014; accepted 10 April 2014 ) 